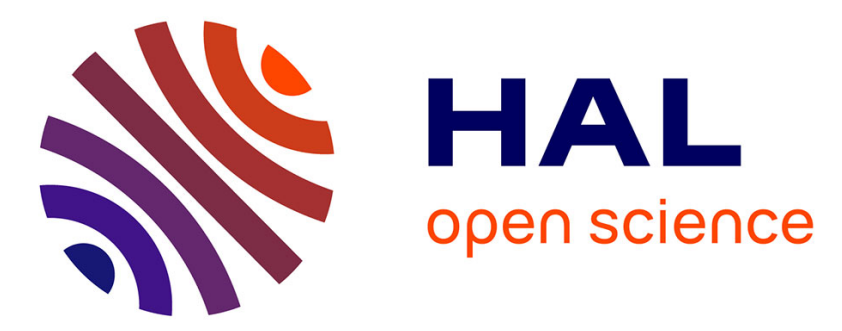

\title{
Former les futurs professionnels de la petite enfance. Entre soin et éducation, quelle place pour les affects?
} Anne-Lise Ulmann, Daniela Rodriguez, Marc Guyon

\section{To cite this version:}

Anne-Lise Ulmann, Daniela Rodriguez, Marc Guyon. Former les futurs professionnels de la petite enfance. Entre soin et éducation, quelle place pour les affects?. Revue des politiques sociales et familiales, 2015, vol. 120 ( $\left.\mathrm{n}^{\circ} 1\right)$, pp. 31-43. 10.3406/caf.2015.3112 . hal-02373000

\section{HAL Id: hal-02373000 \\ https://hal-cnam.archives-ouvertes.fr/hal-02373000}

Submitted on 20 Nov 2019

HAL is a multi-disciplinary open access archive for the deposit and dissemination of scientific research documents, whether they are published or not. The documents may come from teaching and research institutions in France or abroad, or from public or private research centers.
L'archive ouverte pluridisciplinaire HAL, est destinée au dépôt et à la diffusion de documents scientifiques de niveau recherche, publiés ou non, émanant des établissements d'enseignement et de recherche français ou étrangers, des laboratoires publics ou privés.

\section{(1) (1) $\$$}

Distributed under a Creative Commons Attribution - NonCommercial - NoDerivatives 44.0 
Former les futurs professionnels de la petite enfance. Entre soin et éducation, quelle place pour les affects?

Anne-Lise Ulmann, Daniela Rodriguez, Marc Guyon

\section{Citer ce document / Cite this document :}

Ulmann Anne-Lise, Rodriguez Daniela, Guyon Marc. Former les futurs professionnels de la petite enfance. Entre soin et éducation, quelle place pour les affects ?. In: Revue des politiques sociales et familiales, $\mathrm{n}^{\circ} 120,2015$. pp. 31-43;

doi : 10.3406/caf.2015.3112

http://www.persee.fr/doc/caf_2101-8081_2015_num_120_1_3112

Document généré le 27/04/2017 


\title{
Résumé
}

À partir d'une recherche ethnographique sur le travail auprès des jeunes enfants et la formation des futurs professionnels de la petite enfance (titulaires d'un certificat d"aptitude professionnelle et auxiliaires de puériculture), effectuée dans des espaces de formation comme dans des lieux d'accueil des enfants (crèches et écoles maternelles), cet article présente la manière dont la formation structure les pratiques professionnelles qui se distinguent des pratiques domestiques. II montre que, pour revaloriser ce travail méconnu, les formateurs substituent aux pratiques profanes des futurs professionnels des techniques de travail élaborées en référence au monde du soin. Des disjonctions entre la formation et l'exercice professionnel visent à mieux faire reconnaître ces métiers mais soulignent la fragilité de ces fondements qui peuvent rendre le travail encore plus difficile à tenir. Les efforts et les difficultés éprouvées pour mettre en pratique des apprentissages visent à juguler la place des affects dans les rapports aux tout-petits.

\begin{abstract}
Training future early childhood professionals

Between care and education, what place is there for emotion?

Based on an ethnographic study of those who work with young children and the training of future early childhood professionals (holders of certificates of professional competence and childcare assistants), which was carried out in training institutions and in childcare facilities (crèches and primary schools), this article demonstrates that how people are trained influences professional practices, as distinct from untrained, non-professional practices. The study shows that to reassert the value of this generally overlooked area of work, trainers replace the practices of untrained future childcare professionals with work techniques created with reference to the caring professions. The discordance between training and what happens in professional practice comes about as a result of the effort to make this area of work better known and respected, but also highlights how fragile its foundations are, which can make the job even more difficult. The efforts made and difficulties encountered in putting into practice what has been learnt in training reflect an attempt to limit the place of emotion in relations with young children.
\end{abstract}




\section{Former les futurs professionnels de la petite enfance}

\section{Entre soin et éducation, quelle place pour les affects?}

\author{
Anne-Lise Ulmann \\ Daniela Rodriguez \\ Marc Guyon
}

\author{
Conservatoire national des arts et métiers (Cnam), Centre de recherche \\ sur le travail et le développement (CRTD) - Équipe d'accueil (EA) 4132. \\ Cnam, Centre de recherche sur la formation (CRF) - EA 1410. \\ Cnam, CRTD - EA 4132.
}

\author{
Mots-clés \\ - Formation \\ - Certificat d'aptitude \\ professionnelle (CAP) \\ - Auxiliaire \\ de puériculture \\ - Pratique professionnelle \\ - Affect \\ - Soin
}

\begin{abstract}
Les métiers de la petite enfance font, depuis une vingtaine d'années, notamment sous l'influence grandissante des théories du « care » (qu'on pourrait traduire par « aide », « soin aux autres »...) (Tronto, 1993 ; Paperman et Laugier, 2006), l'objet de questionnements multiples pour tenter de désenclaver ces activités des tâches domestiques dans lesquelles elles se trouvent enserrées (Laufer et al., 2003 ; Cresson et Gadrey, 2004 ; Murcier, 2007). Si ces études s'emploient à invalider l'analogie avec les tâches domestiques et à dénoncer les fréquentes assignations de genre, elles influent peu sur les pratiques d'orientation : ces métiers sont essentiellement destinés aux jeunes filles en difficulté scolaire ou à des femmes en reprise tardive d'études, souvent d'origine étrangère et avec un petit niveau d'études (1). Prévaut donc toujours implicitement l'idée que ce type d'activité convient mieux aux femmes (qui composent plus de $90 \%$ de cette population) qu'aux hommes (Maruani et Meron, 2012 ; Murcier, 2004), et qu'à défaut de compétences, des qualités peuvent suffire pour les exercer, du moins pour les professionnels qui sont les moins qualifiés ${ }^{(2)}$, titulaires du certificat $d^{\prime}$ aptitude professionnelle petite enfance (CAP PE) et auxiliaires de puériculture (Ulmann et al., 2011).
\end{abstract}

$C^{\prime}$ est donc dans un contexte en tension entre reconnaissance de la professionnalité et reproduction de certains modèles familiaux où des " capacités naturelles » sont supposées aux femmes (Laufer et al., 2003), que les professionnels de la formation vont apprendre à des jeunes femmes, orientées sur cette voie souvent par défaut, à faire ce travail auprès des enfants. En ce qui concerne la reconnaissance d'une professionnalité, les politiques de la " petite enfance " s'intéressent principalement aux statuts de ces professionnelles (notamment ceux des assistantes maternelles particulièrement précaires) et à leur rémunération (Fagnani, 2001). De nombreuses études s'intéressent à la «professionnalisation » (Aballéa, 2005 ;
Bouve et Sellenet, 2011) mais, plus centrées sur l'emploi que sur le travail, elles laissent dans l'ombre la manière dont la formation contribue à organiser et structurer les manières d'agir et de penser le travail auprès des enfants.

Cet article explore donc les liens entre le travail et la formation pour ces futurs professionnels en adoptant volontairement une focale resserrée sur les conduites des formateurs et des professionnels des crèches pour faire apprendre le métier. Si les tensions éprouvées par les professionnels qui exercent ces métiers sont désormais mieux connues (Cresson, 1998 ; Rayna, 2011 ; Marchand Montanaro, 2013), peu de recherches s'intéressent à la manière dont la formation contribue à structurer les modes d'organisation de l'action en situation réelle de travail. Comment les professionnels se trouvent-ils préparés à faire face à ce travail quotidien, traversé de petits embarras, anodins en apparence, mais essentiel pour la qualité de I'accueil des enfants ? Qu'apprennent-ils à faire en formation qui leur permette d'être reconnus comme " de vrais professionnels » qui connaissent leur métier et n'agissent pas seulement par intuition ou habitude? S'agissant de métiers dont les compétences sont fréquemment assimilées aux pratiques domestiques, ces questions sont centrales. Elles obligent à s'intéresser à la manière dont la formation fait ressource pour développer une professionnalité qui peut se différencier des qualités maternantes toujours très présentes dans les représentations de ce travail. Elles viennent également éclairer la manière dont les apprentissages peuvent contribuer à davantage de reconnaissance pour les professionnels qui exercent ces métiers. Ce questionnement fonde l'approche méthodologique où l'on cherche à appréhender comment les situations de formation et les situations de travail contribuent à construire les compétences mises en œuvre dans le travail auprès des enfants.

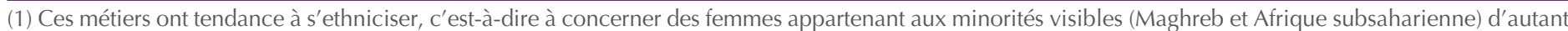
que ces populations sont souvent assignées à des dispositions maternantes.

(2) Ces remarques ne concernent pas les éducateurs de jeunes enfants (EJE) dont le niveau d'études est supérieur au baccalauréat.

Revue des politiques sociales et familiales $n^{\circ} 120-2^{e}$ trimestre 2015

31 Dossier " Accueil du jeune enfant » 
Le travail d'analyse a été réalisé à partir d'une double démarche de type ethnographique dans des structures d'accueil collectif (crèches) et dans les espaces de formation pendant dix-huit mois. L'approche visait à saisir le travail en train de se faire, tant du point de vue de la formation pour appréhender les situations d'apprentissage préparant à ces activités professionnelles que du point de vue de l'activité auprès des enfants, pour comprendre le travail tel qu'il se fait et se donne à voir à de futurs professionnels.

L'ensemble des observations et des entretiens porte sur trois structures d'accueil (deux crèches municipales, et une crèche parentale), ainsi que sur trois établissements de formation - un centre de formation d'apprentis (CFA) et deux lycées professionnels (dont l'un abrite aussi un CFA) - qui assurent des formations à ces deux qualifications selon plusieurs modalités : en formation initiale avec des stages, en alternance avec un contrat d'apprentissage et en formation continue. Ont particulièrement été suivis deux enseignants dans chaque établissement scolaire et quatre d'entre eux dans le centre d'apprentissage (CFA). Les observations ont porté sur plusieurs types d'activité : les enseignements professionnels mais aussi les visites des stagiaires dans les structures d'accueil, les retours de stages et les évaluations en situation de travail. Ces observations ont été complétées par des entretiens permettant d'aborder plus largement les conceptions du travail des stagiaires et leurs trajectoires professionnelles.

Dans les structures d'accueil des enfants, essentiellement les crèches, le travail des équipes intervenant auprès des enfants a été observé, surtout les fonctions de tuteurs. Dans ces structures, des entretiens individuels ont également été réalisés avec les directrices et les adjointes sur leur organisation collective et les difficultés rencontrées avec les professionnels débutants.

Concernant les écoles maternelles il n'a pas été possible d'effectuer d'observations du travail, si ce n'est sur des temps très courts. Mais des entretiens ont pu être conduits auprès des directrices qui recevaient les stagiaires ainsi que l'examen des situations d'évaluation des stages qui s'effectuent entre les tuteurs, la directrice et la formatrice du centre de formation. Ces situations ont permis d'appréhender indirectement le travail demandé à ces futurs professionnels et les décalages qu'ils pouvaient éprouver au regard des activités effectuées en formation.

Dans les centres de formation comme dans les crèches, les entretiens et les observations ont été menés de manière non impliquée, mais l'immersion sur un temps long a permis de nouer des liens qui ont contribué à élargir la compréhension du travail, notamment en permettant à l'équipe de recherche d'avoir accès à différentes réunions internes portant sur I'organisation du travail ou sur la régulation des groupes dans le cours de la formation. Les réunions de travail collectif ont été l'occasion de mettre en débat les premières analyses.

Cette recherche, qui a bénéficié d'un financement de la Caisse Nationale des Allocations familiales dans le cadre d'un appel à projets de l'Observatoire national de la petite enfance sur la qualité de l'accueil des jeunes enfants, porte volontairement sur peu d'établissements. Elle ne vise pas à dégager une connaissance exhaustive des modalités d'apprentissage mais questionne plutôt la manière dont les débutants à ces fonctions structurent une compréhension de leur métier à partir des apports de la formation et de la réalité du travail auprès des enfants. Les situations de travail évoquées constituent des appuis pour appréhender plus concrètement ce que font les professionnels pour orienter, conduire, ajuster leurs actions auprès des enfants. Elles permettent ainsi de saisir une éventuelle dissonance perçue dans le cours de l'activité entre le travail auprès des enfants et le travail appris en formation. À partir de ces dissonances qui se manifestent par des imprévus (un enfant qui mord...), des situations délicates (contenir un groupe d'enfants agités...) ou surprenantes (des demandes particulières de parents...), on peut interroger I'organisation du travail formelle à laquelle s'articule l'organisation réelle du travail (Dejours, 2009). C'est donc comme révélateurs de la complexité de cette professionnalité, qui concerne de nombreux métiers de la relation, que ces dissonances constituent des ressources pour éclairer certaines dimensions du métier, difficiles à expliciter et qui, de ce fait, restent peu visibles ou non dites.

Comprendre la manière dont la formation opère dans la valorisation de ces métiers nécessite tout d'abord d'appréhender les quelques grands principes qui structurent I'organisation des parcours des CAP et des auxiliaires. L'article montrera que ces deux qualifications sont porteuses d'une histoire professionnelle qui organise des trajectoires d'apprentissages, aujourd'hui en changement, mais néanmoins très structurées dans les relations sociales des diplômes relevant de ce champ. L'attention sera ensuite portée sur les liens et les tensions repérés entre la formation et le travail, notamment la manière dont la formation est investie par les formateurs et les professionnels des structures d'accueil pour valoriser l'activité à partir de savoirs appartenant essentiellement au champ du soin. Les implicites et les silences qui structurent le travail seront enfin questionnés.

\section{Des lignes de fracture entre l'emploi et la formation}

Le CAP PE et le diplôme d'auxiliaire de puériculture sont deux qualifications de niveau $\mathrm{V}$ qui préparent aux métiers de la petite enfance pour des personnes qui ont souvent été orientées sur cette voie de manière plutôt subie que choisie, et qui sont essentiellement en attente d'accès à 
un emploi (Odena, 2009, Cresson, 1998) (encadrés, p. 32 et 38). Les deux formations ont une même visée professionnalisante puisqu'il s'agit de permettre aux futurs professionnels d'apprendre le métier non seulement par des cours, mais également par son exercice pratique. Les périodes de stages sont effectuées dans différents types de structure de la petite enfance : crèches, haltes-garderies, relais maternels... Pour le CAP, la structure privilégiée est I'école maternelle tandis que la formation des auxiliaires s'effectue principalement en crèche avec l'obligation de réaliser au moins un stage (très valorisé d'ailleurs) dans le milieu hospitalier : maternités, services accueillant des enfants malades ou porteurs de handicap, structures d'aide sociale à l'enfance. Outre le concours, c'est également par la différence des espaces vécus dans le cadre des stages que la formation va contribuer à construire deux profils professionnels : la place des CAP PE est celle d'appui au travail des enseignants tandis que la place des auxiliaires est celle d'appui au travail des puéricultrices en milieu hospitalier et en crèche.

D'un point de vue historique, le CAP PE a été créé en 1991 pour donner une qualification aux agents travaillant en école maternelle. À présent, $60 \%$ des postes ouverts à concours externe pour devenir agent territorial spécialisé d'école maternelle (Atsem) sont destinés aux titulaires du CAP PE (décret du 13 février 2007). Si dans le plan d'études il est précisé que les détenteurs du CAP PE ont le droit d'exercer leurs fonctions dans « tout établissement accueillant des enfants entre 0 et 6 ans », ces professionnels sont principalement destinés à travailler chez des particuliers ou dans les écoles maternelles. Deux raisons peuvent être avancées : les origines historiques du diplôme et le ministère certificateur (Éducation nationale). En effet, le CAP PE est un diplôme dédié au travail avec les jeunes enfants dans les structures d'accueil collectif, mais il peine à être reconnu pour ce type de travail (3) dans les crèches. Le diplôme d'auxiliaire a un spectre d'intervention plus large puisqu'il concerne également les structu res hospitalières (4) (et les enfants au-delà de 6 ans); il est valorisé pour ces fonctions dans les crèches ce qui confirme l'importance du rôle du ministère certificateur qui régule le fonctionnement de ces structures (Code de la santé publique). Cette situation porte à conséquence dans l'espace professionnel où il est possible de repérer des tensions, depuis la blessure individuelle imperceptible parfois jusqu'au conflit entre les professionnels.

La manière de nommer les professionnels constitue un des indicateurs de la différence de reconnaissance. Les CAP PE dans les crèches sont considérés et désignés comme "personnels non diplômés ». La blessure est $d^{\prime}$ autant plus forte que bien des structures, contraintes financièrement, engagent effectivement des personnels sans aucune qualification (donc sans le CAP). Certaines crèches, pour amoindrir cette identification négative, les appellent "les berceuses », ce qui ne contribue pas à la reconnaissance du travail réellement effectué, voire peut le déprécier davantage en le maintenant dans l'ombre. Le diplôme CAP PE, malgré le travail effectué en formation avec les futurs professionnels, ne semble pas reconnu par les directions des structures, qui estiment les titulaires de ce diplôme moins bien formés que les auxiliaires de puériculture. Pour les titulaires du CAP PE, l'investissement dans I'apprentissage se trouve donc peu payé en retour : "Nous, de toutes les manières, on n'est rien; on a notre CAP mais ça ne fait rien, pour eux [les personnels « qualifiés » de la crèche] on n'est rien... ». Si, comme le rappelle Danièle Kergoat, "pour pouvoir se dire par un nom de métier il est nécessaire de pouvoir penser ce métier dans un certaine durée » (1995, p. 171), ce défaut de nomination constitue sans doute une entrave supplémentaire pour faire valoir sa place au sein des équipes. Sans nom, ces professionnels semblent assignés à davantage d'invisibilité sociale que les auxiliaires de puériculture.

On peut donc faire l'hypothèse qu'une partie des tensions entre ces différentes catégories de personnels [éducateurs de jeunes enfants (EJE), auxiliaires de puériculture, CAP PE et, parfois, personnels sans aucun diplôme], qui sont destinés à œuvrer ensemble dans les structures d'accueil collectif, a sa source dans l'organisation même de ces cursus de formation. Cette organisation est liée à la présence de différentes tutelles, ce qui met en opposition ou en situation d'ignorance mutuelle le point de vue médical et le point de vue éducatif. Le point de vue médical - dont le diplôme d'auxiliaire de puériculture serait le signe et les puéricultrices, les représentantes prime dans les crèches. Les détenteurs du CAP PE comprennent, sur le terrain, qu'ils ne sont pas les bienvenus

\footnotetext{
(3) Certaines municipalités (mais pas toutes), par souci de la qualification des professionnels et sans doute aussi parce que le ministère de tutelle correspond mieux aux représentations de la crèche, n'autorisent pas que les titulaires du CAP interviennent directement auprès des enfants dans les crèches. Ces professionnels restent alors cantonnés aux fonctions logistiques de la crèche : préparation des repas, ménage...

(4) Si la formation des auxiliaires intègre un exercice possible, très prisé, de la fonction en milieu hospitalier, il semble que, dans les hôpitaux et les maternités, les auxiliaires soient de moins en moins recherchées au profit des aides-soignantes polyvalentes. L'insertion professionnelle dans une crèche apparaît souvent comme un déclassement au regard des ambitions initiales.
}

Revue des politiques sociales et familiales $n^{\circ} 120$ - 2e trimestre 2015

33 Dossier " Accueil du jeune enfant » 
dans les crèches, malgré les promesses faites par la formation. Des clivages de même type existent également (même s'ils ne sont pas étudiés ici) entre les EJE, promoteurs de la dimension socioéducative dans les crèches, et les puéricultrices, qui représentent la dimension sanitaire.

Comme les tutelles ne coopèrent pas pour concevoir les maquettes pédagogiques de ces diplômes, les formateurs qui animent ces formations sont amenés à reproduire les lignes de fracture entre les uns et les autres. En confortant les statuts différenciés et hiérarchisés ils contribuent à installer des rapports de concurrence entre les professionnels, ce qui peut éclairer, autrement que par le genre, les conflits dans les équipes, fréquemment notés par les directions de crèche : " ce sont des milieux de femmes et c'est difficile, un rien peut déclencher des histoires, elles sont toute la journée ensemble dans la même pièce à regarder ce que fait l'autre... » (directrice de crèche).

\section{Les pratiques profanes à l'épreuve des apprentissages : I'emprise du soin}

Comme toute formation, I'apprentissage des règles et des exigences du métier suppose que les personnes en formation acceptent une certaine ouverture à de nouveaux savoirs pour en faire usage dans l'exercice de leur futur métier. Cette soumission aux savoirs nouveaux est une condition importante de l'obtention d'une qualification donnant accès à un corps de métier constitué. L'apprentissage des règles auxquelles se conformer est délicat parce que ce qui doit être appris peut être perçu par les futurs professionnels soit comme déjà su en raison d'une expérience du travail domestique, soit comme inaccessible voire non valide, en raison de traditions sociales et culturelles différentes de celles véhiculées par la formation. Que faire apprendre en formation qui puisse à la fois avoir du sens pour les personnes qui vont exercer ce travail, être utile au travail en train de se faire et contribuer à revaloriser l'image de ces métiers ? Comment et sur quoi différencier l'activité professionnelle de l'activité domestique quand elles se superposent, au point de se confondre, et qu'elles portent sur le même objet, l'enfant ? Une des réponses apportées à ces questions par les formateurs sera l'apprentissage d'un corpus de savoirs considérés comme scientifiques, donc difficiles à contester et permettant de neutraliser les savoirs des expériences antérieures.

\section{L'apprentissage d'une langue d'emprunt issue du monde du soin}

Parmi les nombreux apprentissages, le langage paraît important à relever. Non seulement il contribue à déplacer le travail avec les enfants de la sphère domestique vers la sphère professionnelle, en opérant une rationalisation des actes, mais il doit aussi permettre de les abriter dans I'orbite du milieu médical. Les futurs professionnels (CAP PE comme auxiliaires de puériculture) acquièrent donc, en formation, une certaine manière de parler. Cet apprentissage langagier ne ressemble ni à un langage opératif (Falzon, 1987), procédant par mots simples pour faciliter l'action, ni à une langue de métier en ce qu'il ne concentre aucune symbolique, il ne permet pas de marquer des liens entre professionnels et il n'est pas lié à l'expression de sentiments éprouvés dans I'agir professionnel (Cru, 1995). Il sert simplement à marquer la distance avec I'univers domestique sans apporter de nouvelles dimensions à l'activité professionnelle. Dans des travaux pratiques de cuisine où il s'agit de confectionner un plat pour un enfant, la formatrice présentera ainsi le travail : « je vous distribue le protocole, vous devez le suivre exactement ». À la lecture du document, on comprend que le "protocole» est un synonyme de « recette » de cuisine, dont le mot n'a jamais été employé au cours de cet enseignement. Cette langue de la formation ne constitue pas simplement un système d'équivalences entre langue profane et langue savante, elle permet de projeter l'activité dans le monde médical pour amener les futurs professionnels à penser leurs actions en fonction du paradigme du soin. Ainsi ne sera-t-il jamais question, en formation comme dans les crèches, de la "relation » ou de la " communication » avec les parents, mais de la "transmission aux parents", qui semble être une analogie au travail infirmier lors du relais d'informations entre les équipes de professionnels.

Les mots n'étant jamais que des mots mais des « programmes d'action »(Gardin, 1995), le travail avec les enfants se trouve ainsi enserré dans l'univers médical et métamorphosé en un "soin », rassemblant dans un vaste ensemble les actions liées à l'hygiène (les changes des enfants, la préparation de leurs biberons...) et celles relevant du champ éducatif (les activités d'éveil...). Dans les discours des formateurs, ces deux types d'action - I'hygiène et les jeux - visant les mêmes buts, le développement de l'enfant et "son bien-être » constituent donc des soins. "Pour un-e professionnel-le, un change, un repas ou une histoire, c'est toujours essayer de répondre au besoin de l'enfant, c'est un soin " (formatrice des auxiliaires). Cette emprise du soin sur l'éducatif installe une différence entre le travail de ces professionnels et celui des EJE, plus clairement, semble-t-il, positionnés sur l'éducatif (Verba, 2014 ; Zogmal, 2008).

L'objectif de ce glissement, qui tend à faire de l'enfant un sujet (ou un objet ?) de soins, est également de rompre

Revue des politiques sociales et familiales $n^{\circ} 120$ - $2^{\text {e }}$ trimestre 2015

34 Dossier "Accueil du jeune enfant » 
avec des mots blessants fréquemment tenus à propos de ces professionnels, comme, par exemple, celui qui est rapporté à propos de la visite d'une personnalité politique dans la crèche qui s'extasie sur ces : « jolis métiers où l'on joue tout le temps... ». II contribue sans doute à l'installation d'une représentation du travail comme un " vrai » métier qui possède sa grammaire langagière et gestuelle mais il peut aussi conduire à une certaine déception lors de la rencontre avec le réel de ce travail. Cette langue d'emprunt, construite à partir de termes appartenant au monde médical, constitue une langue de travail qui n'a d'usages véritables que dans l'espace de la formation, pour donner un peu de prestige à des activités trop souvent rabattues à un travail domestique sans savoirs. Cette langue installe néanmoins une disjonction entre le travail réel et son apprentissage et, tout en contribuant à valoriser I'activité, construit la professionnalité sur des fondements fragiles. Si la technicisation des actes de métiers est un processus fréquent permettant de les extraire de la banalité quotidienne, elle frappe ici par sa centralité dans les apprentissages et le déni qu'elle peut entraîner sur la prise en compte des expériences antérieures, centrales pour développer de nouvelles pratiques professionnelles.

\section{L'apprentissage d'une posture professionnelle par des techniques gestuelles}

Si la formation professionnelle suppose autant d'apprendre que de désapprendre pour s'approprier d'autres manières d'agir (par exemple, pour protéger le corps d'une trop forte exposition à des risques, ou pour élaborer des raisonnements prenant en compte plus de dimensions), elle vise généralement un objectif, c'est-à-dire un résultat pour I'activité en lien avec l'apprentissage. Comme le rappelle Jacques Leplat, " I'opérateur qui apprend la tâche n'apprend pas une séquence de gestes mais le but fixé par la tâche » (2013, p. 12). Dans les formations observées, la focalisation des enseignements sur des gestes particuliers vise, comme pour l'apprentissage d'une langue d'emprunt, principalement à se différencier des pratiques domestiques. Ils sont donc des «signes » (Leplat) de cette capacité à prendre des distances avec des pratiques profanes, à faire la part entre la « maison » et la crèche. On repère, dans la formation, que ces entraînements aux gestes professionnels peuvent avoir deux fonctions : ils contribuent à mettre en scène une dextérité, une habileté à faire et fonctionnent comme signes de l'appartenance professionnelle ; mais ils apprennent également une certaine posture distancée à l'enfant et constituent, dès lors, un moyen pour conformer ces futurs professionnels aux codes sociaux et moraux que cherche à transmettre la formation.
Le geste comme signe de I'appartenance professionnelle

La formation s'attache également à faire apprendre des gestuelles permettant de savoir agir avec une certaine dextérité en mobilisant son corps et le corps de l'autre au moyen de techniques et de méthodes manifestement contre-intuitives. Le nettoyage des matelas de change des enfants, par exemple, doit se faire d'une certaine manière : "Non! Ce n'est pas ce qu'on a appris ! Rappelez-vous, d'abord [fait le geste en même temps qu'elle le dit], on passe le chiffon de l'extérieur vers l'intérieur pour les côtés et après, dans le cadre que l'on vient de faire, de gauche à droite, comme ça, jusqu'au bout. Ça [mime le geste effectué par la jeune fille], c'est à la maison, jamais à la crèche! » (formatrice CAP PE).

Beaucoup de travaux pratiques sont donc consacrés à des entraînements de ce type : le lavage de mains, imité des techniques pratiquées par les chirurgiens, le ramassage de saletés sur le sol en les enroulant dans la serpillière sans recourir à une pelle à ordures, les changes des couches, le portage des enfants... Ces entraînements obligent les apprenants à désapprendre ce qu'ils peuvent faire spontanément pour s'entraîner à la maîtrise de ces nouvelles techniques. Le change des couches des enfants est fréquemment évoqué par les professionnels comme particulièrement délicat parce qu'il entre en concurrence avec les conduites des parents : "Changer en retournant le bébé sur la table sans lui lever les jambes, juste en le retournant, je trouve ça difficile... En plus, à la maison, les parents les soulèvent, alors ils prennent l'habitude et, dès qu'on les pose sur la table, souvent ils lèvent les jambes tout seuls » (apprentie auxiliaire de puériculture). Cette apprentie auxiliaire, aux prises simultanément avec l'exigence professionnelle et le respect des pratiques parentales manifestées par le comportement spontané de l'enfant, exprime les tensions entre plusieurs sources de normes : celles de sa culture, de sa famille, de ses valeurs... qui se heurtent à celles de la formation qui impose d'autres pratiques. Les "débats de normes » (Schwartz, 1988) que ces différences pourraient occasionner sont écartés en formation pour imposer des principes de travail qui ont pour origine les savoirs de la médecine.

L'enjeu du geste comme signe d'une appartenance à un corps de métier est tel que, même à propos d'une image sur un livre de travaux pratiques, une formatrice dira, irritée, au chercheur : "Là je trouve que c'est vraiment un problème. C'est le contraire de tout ce qu'on leur apprend. On ne change pas un enfant comme cela! Les fesses en l'air en le prenant par les pieds ! Ils font cela à

Revue des politiques sociales et familiales $n^{\circ} 120$ - 2e trimestre 2015 
I'examen, c'est sûr ils sont recalés ! En plus c'est vraiment un non-respect de la pudeur de l'enfant... Pour moi, je trouve que c'est de la maltraitance, et travailler avec cela c'est... Ce livre me pose vraiment problème parce qu'il y a de nombreux passages comme celui-ci que je ne peux pas utiliser » (formatrice CAP PE).

\section{Le geste comme moyen d'une conformation aux codes sociaux et moraux}

D'autres gestes, qui vont concerner le portage d'un enfant ou la manière de lui donner un biberon, vont apprendre à installer une distance relationnelle à l'enfant en technicisant l'engagement dans la manière $d$ 'interagir avec lui. L'objectif de cet apprentissage technique est de limiter l'engagement corporel du professionnel dans cette relation à l'enfant. De ce point de vue, la maîtrise technique produirait de facto la distance juste, le bon acte professionnel, en faisant l'économie de débats sur les affects dans le travail avec les tout-petits. "Certaines ont du mal avec le fait que les enfants ne sont pas leurs objets. Elles les portent en les serrant contre elles, là, comme leurs choses à elles, tout proche comme ça! [imite le portage]. Mais ce n'est pas ça, bien porter un bébé. II faut lui maintenir le dos, la tête, qu'il se sente contenu mais le décoller de son corps pour le laisser libre de ses mouvements. Tant qu'elles ne savent pas mettre cette distance, à mon avis, elles ne peuvent pas travailler avec les bébés, elles sont trop dans l'affectif, dans la possession, et ce n'est pas notre rôle » (adjointe de direction d'une crèche).

Ces propos concernant des apprenties accueillies dans la crèche montrent que les techniques gestuelles sont bien en lien avec les buts de l'action, mais ces derniers concernent autant la situation professionnelle (porter l'enfant sans l'entraver dans ses mouvements) que la manière dont le professionnel s'y engage (ne pas être "dans l'affectif »), son activité. L'apprentissage du geste sert à installer une distance, à canaliser les affects dans des situations où ils pourraient se manifester trop fortement. Peut-on faire I'hypothèse que cet apprentissage technique, sans lien avec les buts de l'activité, permet d'euphémiser voire de contourner la question de la place des affects dans le travail avec les enfants ? De ce point de vue, le geste semble aussi servir à faire apprendre le respect de codes sociaux et moraux en limitant les échanges sur les savoirs et les craintes qui les fondent. Le geste serait la manifestation externe d'une certaine éthique professionnelle qu'il faudrait respecter pour ne pas se trouver en situation de faute. Ainsi, lors d'un retour de stage où l'apprentie auxiliaire rapporte ses difficultés à changer la couche d'un enfant en effectuant le geste appris sur les poupons, la formatrice répond : " je sais, c'est difficile mais c'est important de faire comme ça [le geste appris], parce que ça ne casse pas le dos en deux, donc pour le confort du bébé c'est mieux [...] ». Dans une autre session de formation, une formatrice explique, à propos de ce même geste, son importance en le comparant au geste profane, qu'elle décrit ainsi : " ce n'est pas beau ni confortable [pour le bébé sur la table à langer] d'être les quatre pattes en l'air ». Ce geste professionnel du change semble s'imposer doublement : pour des raisons médicales (dont les explications resteront sommaires mais impératives), et pour des raisons morales et esthétiques. Implicitement, il est donc dit à ces futurs professionnels que céder à la facilité en ne changeant pas l'enfant selon les règles de cette professionnalité construite pourrait relever d'une faute au sens où cette manière $d^{\prime}$ agir ne serait pas bonne pour le destinataire du soin.

Cet apprentissage technique, qui occupe une part importante des heures de formation, a comme effet de limiter les échanges sur une autre dimension moins technique du métier, mais néanmoins très présente et peu évoquée : le sexe des enfants. À propos d'un jeune homme débutant en CAP PE, les professionnelles le testent lors de son stage en crèche sur son rapport au sexe d'une petite fille et rapportent, en riant, "On lui a même demandé de changer une petite fille, pour voir, mais [rires] il s'en est bien sorti... ! ». Rejetées dans l'évidence ou le naturel, les questions relatives au sexe des enfants n'ont pas davantage de place en formation que dans les échanges entre professionnels, si ce n'est sous la forme de plaisanteries ou de règles de travail : "ne pas être aux toilettes avec les enfants, mais les attendre derrière la porte », " ne pas nettoyer les enfants mais les laisser faire le plus possible eux-mêmes » (formatrice CAP PE et tutrice d'école maternelle). Dissociant clairement le sexe de la sexualité, les formateurs concentrent les apprentissages sur des techniques et évitent ces sujets qui semblent pourtant très présents si l'on accorde de l'importance à leurs manifestations indirectes. Ainsi, dans un cours sur les techniques de change, face à des jeunes filles qui s'amusaient de manière très visible à dire qu'elles jouaient "à un couple de lesbiennes qui changent leur enfant », la formatrice ne relèvera pas la situation et continuera son enseignement. L'apprentissage du geste paraît un moyen pour protéger l'espace de la formation de débats professionnels comme ceux rapportés par la jeune fille sur sa situation en stage, et rabattre les dimensions éthiques du travail sur I'acquisition de techniques. 
Extrait d'un échange entre une formatrice et une apprentie CAP PE

lors d'une séance de retour de stage

- "J'ai dû changer une petite qui avait fait pipi dans sa culotte, mais vous dites qu'il faut laver les enfants, mais pas du tout!»

- "Comment ça pas du tout ! Si la petite a fait pipi c'est quand même mieux de la passer un peu à l'eau avant de lui remettre du linge propre ».

- "Et bien non ! Ma tutrice m'a dit que je n'avais pas à faire ça. Que c'était même une faute parce qu'on pouvait croire que... je ne sais pas quoi, que je la touche, et tout... " - " mais bien sûr! Parce qu'elle a l'âge de se laver seule. Mais vous lui dites, rince-toi avant de remettre ta culotte. Elle peut le faire seule bien sûr ! »

- « oui, ben non, même ça c'est non. La consigne c'est $q u$ 'elle remet un slip propre à elle et puis voilà on la ramène dans la classe ».

Les gestes à apprendre sont nombreux et semblent presque toujours exiger des professionnels qu'ils se conforment aux normes d'une professionnalité qui visent à se différencier de pratiques profanes en prenant fréquemment pour référence l'univers médical. L'apprentissage du métier semble se concentrer à la fois sur des « gestes signes » en ce qu'ils peuvent donner à voir une différence avec le fonctionnement profane (notamment celui de parent) et sur des savoirs médicaux, éventuellement réinterprétés (Boltanski,1971), mais non discutables. S'adressant à de jeunes professionnels, cet apprentissage des techniques gestuelles apprend plus que le geste ; il conforme à un rapport soumis à l'autorité de ceux qui détiennent le savoir et, dans le même temps, suppose leur adhésion à un certain ordre du monde. À propos d'une auxiliaire, pourtant confirmée dans la fonction, une directrice de crèche explique : " elle a de très bons rapports avec les enfants, mais alors, tout ce qu'elle fait c'est brouillon, ça part dans tous les sens, elle n'est jamais bien posée...». L'apprentissage des règles auxquelles se conformer va au-delà du champ professionnel ; il entraîne également une adhésion à une représentation sociale d'un monde qui doit être propre, ordonné, rangé, organisé, voire quasi stérile pour accueillir les enfants. De cette façon, le bon geste, dans l'ensemble de ses composantes, techniques, sociales et morales, ne peut produire qu'un bon résultat pour l'enfant.

\section{Le travail à l'épreuve de ses tensions : la place des affects}

L'apprentissage du langage et des gestes contribue à valoriser le travail en l'infléchissant vers un univers médicalisé, dégagé d’affects et sans désordre. Cette inflexion permet de tourner le dos à une activité qui serait assimilable à la garde (terme proscrit) pour une pratique qui se veut soignante. Ce soin de la puériculture est néanmoins particulier puisqu'il ne s'adresse pas à des personnes qui seraient malades ou affaiblies mais à des enfants fortement dépendants et en développement psychocorporel. Comme le soin médical, il se fonde sur des principes d'hygiène qui en constituent l'un des socles et des éléments de savoirs scientifiques, mais il se déploie ensuite à partir de conceptions importées de la psychologie du développement et du champ éducatif.

\section{L'observation pour faire écran à la spontanéité}

Dans les deux formations, les formateurs mettent l'accent sur l'importance de savoir observer l'enfant avant d'interagir avec lui. Les premiers stages sont d'ailleurs souvent considérés comme des stages d'observation où les apprentis doivent apprendre à observer le travail pour ne pas " être dans le faire », qui renvoie à l'activité spontanée, mais être dans ce que les formatrices nomment « le savoir-faire" " qui suppose davantage de réflexivité. Cet apprentissage, pour les auxiliaires de puériculture, s'effectue à partir de "la démarche de soins ", méthode importée des soins infirmiers pour mettre en liens des symptômes avec l'étiologie d'une maladie et donner le traitement adapté. Dans cette démarche, les futurs professionnels doivent apprendre à organiser leurs actions à partir $\mathrm{d}^{\prime}$ un tableau où ils inscrivent, en colonnes, leurs observations concernant l'enfant, l'identification de leurs besoins compte tenu de l'observation et leur action justifiée au regard des besoins. Lors d'une démarche de soin, la stagiaire explique à la formatrice et à la tutrice ce qu'elle a observé du déroulement d'un repas :

- Stagiaire : "À 12 h 30 le repas est servi et à 15 h 30 il y a le goûter. Le chef cuisinier prépare un gâteau, un produit laitier. Par exemple, du fromage».

- Formatrice : "Il est important de préciser les quantités dans la fiche ${ }^{(5)}$. On doit analyser les besoins nutritionnels pour un enfant de deux ans ".

La stagiaire essaye de distinguer dans sa feuille ce qui relève de l'observation et de l'analyse.

- La formatrice reprend : "Nous, notre travail en tant que professionnelles est de mettre en lien les observations avec les connaissances pour mettre en place des actions pertinentes et faire aussi de la prévention. Pour réaliser la démarche des soins, il est important d'observer mais aussi de connaître les contenus des cours et les actions concernant la prise en charge de l'enfant ${ }^{(6)}$ ».

(5) La description réalisée est écrite sur une feuille et présentée oralement à la formatrice.

(6) Extrait de l'analyse de la démarche des soins qui a suivi à la réalisation du soin.

Revue des politiques sociales et familiales $n^{\circ} 120$ - $2^{\text {e }}$ trimestre 2015

37 Dossier « Accueil du jeune enfant » 
Le certificat d'aptitude professionnelle petite enfance (CAP PE) et le diplôme d'auxiliaire de puériculture sont deux qualifications de même niveau (niveau V), qui ne bénéficient pas de la même reconnaissance sociale dans le milieu professionnel. Les conditions d'accès pour s'inscrire à ces diplômes et les parcours de vie antérieurs des personnes qui suivent ces formations vont contribuer à ces différences de reconnaissance pour ces deux qualifications. Le CAP PE, diplôme délivré par I'Éducation nationale, est accessible à toute personne au sortir du collège à partir de 16 ans, selon trois modalités : en formation initiale, en alternance avec un contrat d'apprentissage ou dans le cadre de la formation professionnelle continue. Les personnes qui s'inscrivent à ces cursus n'ont pas tout à fait les mêmes profils. En formation initiale, il s'agit, la plupart du temps, d'élèves de collèges orientés sans sélection (et souvent par défaut), vers un lycée professionnel ; sous le statut d'apprenti, pour des jeunes âgés de 16 ans à 25 ans, le nombre de places disponibles dans les centres de formation d'apprentis (CFA), et la nécessité de trouver un employeur pour établir un contrat d'apprentissage, implique de leur part une démarche volontaire pour intégrer la formation, qui est considérée par les responsables pédagogiques comme un gage de motivation pour cette orientation. Enfin, la formation professionnelle continue concerne un public généralement un peu plus âgé en reprise d'études ou en reconversion professionnelle qui manifeste un intérêt plus marqué pour le choix de ce métier.

La formation au diplôme d'auxiliaire de puériculture, certifié par le ministère des Affaires sociales et de la Santé (1), s'effectue également selon ces trois modalités (initiale, alternance et formation continue) mais, à la différence du CAP PE, elle suppose, pour y être admis, la réussite à un concours (arrêté du 15 mars 2010). Aucune condition de diplôme n'est requise pour se présenter à l'épreuve écrite d'admissibilité du concours. Les candidats déjà titulaires d'un diplôme de niveau V du domaine sanitaire et social (notamment le CAP PE) ou de niveau supérieur sont dispensés de l'épreuve écrite de culture générale d'admissibilité mais doivent passer l'oral de ce concours. Celui-ci se compose de deux épreuves : la première pour tester les capacités d'argumentation et les aptitudes à suivre la formation (tests psychotechniques), la seconde pour « évaluer la motivation du candidat » (arrêté du 16 janvier 2006). Le concours constitue donc un puissant dispositif de valorisation de la formation et du diplôme attirant des personnes ayant un niveau scolaire plus élevé (2).

Ces deux diplômes conduisent à des activités professionnelles, parfois similaires et parfois un peu différentes selon les lieux où elles sont exercées. Le secteur hospitalier est réservé aux auxiliaires et les écoles maternelles aux CAP PE. Cependant, I'analyse des conditions réelles de l'emploi montre que peu d'auxiliaires exercent en milieux hospitaliers et que les CAP PE sont fréquemment employés dans les crèches, à l'exception des crèches publiques de certaines grandes villes comme Paris.

(1) Diplôme enregistré dans le répertoire national des certifications professionnelles.

(2) En 2010, 45 \% des jeunes accédant à ce type de formation ont un niveau inférieur au baccalauréat : 9 \% sont sans qualification et $36 \%$ sont titulaires d'un CAP ou d'un brevet d'études professionnelles ; $51 \%$ déclarent avoir un niveau au moins équivalent au baccalauréat. Source: Observatoire régional emploi formation (Oref, île-de-France, 2010, 69 pages), Métiers de la petite enfance en Île-de-France. Diagnostic partagé de la formation et de l'emploi. Rapport réalisé dans le cadre de l'appui de l'Oref et de la formation d'île-de-France à l'élaboration et au suivi du schéma régional des formations sanitaires et sociales du conseil régional d'île-deFrance (service des formations sanitaires et sociales) - rédacteur : N. Goyaux.

Sans symptôme, ni maladie de l'enfant, l'observation d'une situation ordinaire se révèle délicate et ne peut porter que sur les détails de la vie quotidienne, comme la description du repas. Pour donner sens, dans ce contexte, à ce travail descriptif, deux éléments sont mis en avant par la formatrice. D'une part, la connaissance des cours, considérant que le savoir et l'action sont dans un rapport de réfléchissement mutuel ; d'autre part, un regard préventif, notamment pour identifier d'éventuelles carences nutritionnelles des enfants. Mais de quelle prévention est-il question ? Est-ce une activité de conseil auprès des parents ? Est-ce une capacité à identifier des carences au cas où une telle situation pourrait se produire? Quel est le but d'une telle action dans un contexte où l'enfant est bien portant? Les propos d'une jeune fille nouvellement diplômée éclairent au moins partiellement ce rapport à la prévention : " les parents ne savent pas la nourriture qu'il faut à leurs enfants. Certains ne veulent pas qu'ils mangent de viande ici, à la crèche, mais le soir ils ne compensent pas, alors, nous, on leur dit, on leur explique... »(7).

La prévention évoquée en formation servirait-elle à intervenir indirectement sur le mode de vie des parents en les conformant également à des règles d'hygiène qu'ils ignoreraient, contrairement à eux ? Ces règles nutritionnelles, qui se fondent sur le savoir de la science et qui, de ce point de vue, ont toute leur pertinence, n'auraient-elles pas aussi pour fonction d'assurer un ordre comme les règles $d^{\prime}$ 'hygiène ont $\mathrm{pu}$, au cours du temps, contribuer au "polissage de la conduite » en tissant subtilement les liens entre l'intime et le social ? (Vigarello, 2014, p. 220).

(7) Auxiliaire diplômée depuis cinq mois et embauchée dans une crèche depuis trois mois.

Revue des politiques sociales et familiales $\mathrm{n}^{\circ} 120-2^{\mathrm{e}}$ trimestre 2015

38 Dossier "Accueil du jeune enfant » 
La prévention serait-elle une modalité d'action destinée aux parents pour leur faire reconnaître la professionnalité de ces métiers?

Ce regard préventif requis par la formation pour s'assurer de la santé de l'enfant est en cohérence avec cette posture subjective particulière qui nécessite la rétention des gestes spontanés pour ne focaliser l'activité que sur le développement de l'enfant. Les futurs professionnels apprennent ainsi en formation à se tenir affectivement à distance de l'enfant pour se centrer sur la compréhension de ce qu'il manifeste et limiter leurs actions à l'accompagnement de ses capacités, parfois appelées «compétences ». La retenue de ce que les formatrices appellent « le faire » sert à rendre visible ces progrès de l'enfant et à valoriser indirectement leur contribution dans ce développement. Les maîtres-mots sont I' « autonomie » et I' « accompagnement ». Cette attention au développement de l'activité de l'enfant fait l'objet d'attentions multiples des professionnelles qui doivent apprendre à savoir détecter, dans les jeux et mouvements des enfants, ce que leurs gestes professionnels leur ont permis d'apprendre. Ainsi, quand la professionnelle joue avec un enfant, elle ne doit pas jouer avec lui, c'est-à-dire s'investir dans une relation ludique. Elle doit, au contraire, rester en extériorité pour voir comment accompagner l'enfant à expérimenter par lui-même ce que ce jeu va lui permettre de faire de manière autonome, et lui proposer, en fonction de ce qu'il manifeste, d'autres activités. Il ne faut donc ni faire à la place de l'enfant ni induire un comportement qui ne viendrait pas complètement de lui, mais être en soutien de ses intentions, ce qui suppose, pour le professionnel, de savoir les décrypter et les interpréter. Il est alors requis des professionnels de savoir saisir les moindres gestes des enfants qui seraient significatifs pour objectiver son développement.

Au cours d'une activité consistant à raconter des histoires en les mimant, la directrice observe la situation et explique au chercheur sa compréhension de ce qu'elle voit :

- Directrice : "Regardez ! L'enfant a compris ! On a lu l'histoire, d'abord en la mimant, puis ensuite on l'a racontée uniquement avec le mime ! Là il s'est passé quelque chose!»

(À propos de la professionnelle qui anime cet atelier) : " On va voir si elle l'a vu... Normalement, elle doit le noter et ensuite en parler lors de notre réunion de liaison. Ensuite on peut faire évoluer l'atelier ».
Un peu plus tard, lors d'une réunion interne :

- Directrice : "Alors, est-ce que tu as noté ce qui s'était passé avec l'enfant $X$ quand tu as lu et tu as mimé ?»

- Professionnelle : «Oui !»

- Directrice : "C'est bien, on va voir à le faire systématiquement maintenant », se retournant vers le chercheur : "Vous voyez, elle l'a vu et elle l'a fait!»

Le jeu à la crèche est envisagé comme une activité aux vertus pédagogiques favorisant un certain développement, mais il ne peut constituer une activité relationnelle où seul le plaisir de jouer aurait sa place ; il est donc considéré comme un "soin » au même titre qu'un repas équilibré. $C^{\prime}$ est sur ce point précisément qu'il se différencie du jeu engagé par les parents. Le seul plaisir autorisé dans le jeu en crèche est la satisfaction générée par l'acte professionnel, celle qui relève du travail bien fait permettant de constater les progrès de l'enfant et donnant à voir sa propre contribution au développement de l'autre.

Dans certaines crèches, l'attention portée aux progrès de I'enfant va nécessiter un traçage de son comportement, consigné - souvent par l'EJE - dans des classeurs. Ces fiches individuelles sur le développement des enfants constituent des artefacts pour mettre en visibilité leurs acquisitions grâce aux "soins » des professionnels. Même si la tenue de ces fiches peut apparaître, de l'extérieur, comme une tâche administrative, lourde, et finalement assez éloignée de l'activité réalisée avec les enfants, les professionnels ne s'en plaignent pas. Un tel travail donne une certaine matérialité à des dimensions fugaces ou impalpables de l'activité, et peut constituer un outil de valorisation partagée d'un travail invisible, même pour les professionnels qui le font. Utilisé parfois comme support à une analyse collective de pratiques, il permet, en outre, de valoriser ou d'ajuster les actions entreprises pour contribuer au développement de l'enfant. Ce traçage du développement de l'enfant est révélateur de la tension entre le soin et l'éducatif et des difficultés à faire reconnaître la spécificité de ce travail. D'un côté, il peut être assimilable à un carnet de santé de l'enfant qui consigne les évolutions de son développement en même temps qu'il contrôle les obligations des parents sur des visites médicales obligatoires. De ce point de vue, il relève bien d'une conception de la crèche comme espace de soin et de prévention, qui réfère l'enfant à des normes de comportements que la crèche a mission de surveiller. De I'autre, il peut également être envisagé comme un bulletin scolaire qui, en donnant à voir les " compétences »(8) de

(8) Le terme est fréquemment employé par les formatrices, les directrices et les EJE mais on l'a aussi entendu de la part d'auxiliaires de puériculture.

Revue des politiques sociales et familiales $n^{\circ} 120-2^{\text {e }}$ trimestre 2015

39 Dossier " Accueil du jeune enfant » 
Extrait d'un échange

entre une formatrice et une stagiaire

- F : «Vous avez une relation privilégiée avec C. et dans quatre semaines vous ne serez plus là ».

- $\mathrm{S}$ : "Mais elle réclame les bras...."

- F : «Elle peut être à côté de vous. Elle va être triste sur le moment mais elle va le surmonter. Il faut dire "non" et aller plus loin, il faut dire pourquoi ».

- S: "Mais c'est une petite fille, elle a peur des autres".

- F : "Et avant que vous ne soyez là, comment se débrouillait-elle? Il faut lui faire comprendre, par exemple, l'accompagner à un jeu pour qu'elle vous oublie».

- S : "Aujourd'hui elle a été encore plus dans la demande. C'est sûr que je ne serai plus là et après... "

- F : "Vous pouvez lui dire "je te prends un peu mais après c'est fini". Elle peut faire autre chose au lieu d'être dans vos bras, comme un jeu ».

- S : «Elle joue très bien, elle fait la dînette".

- F: "Alors vous lui dites "Viens on va jouer" et vous I'accompagnez ».

chaque enfant, installe la crèche comme une propédeutique à l'école maternelle.

\section{Distance professionnelle et manifestation des affects : un équilibre délicat}

Si ce rapprochement avec le monde du soin contribue à la reconnaissance de ces deux métiers de la petite enfance, il peut tendre vers une reconfiguration de l'espace de la crèche en un espace soignant, presque stérile, où s'impose parfois le port de vêtements particuliers (notamment des chaussons pour les parents). Cet investissement dans un univers idéal, qui doit être protégé des multiples pollutions du monde extérieur, semble engendrer un système de relations précautionneux qui tient à distance les affects dans les liens établis avec les enfants. L'observance des règles $d^{\prime}$ hyggiène relèverait plus globalement d'un certain ascétisme qui commence par la retenue des gestes spontanés et se prolonge par la mise à distance des affects dans le travail. Le geste bien fait et l'observation distancée du comportement des enfants peuvent donc être interprétés comme des modalités de travail destinées à retenir les affects qui traversent la relation aux enfants.

De manière paradoxale, la question des affects est rarement évoquée comme telle en formation, alors qu'elle est centrale dans la construction de la professionnalité. Cet apprentissage de la distance s'effectue par la pratique de gestes qui technicisent la relation et s'imposent aux apprentis sans que les savoirs qui les fondent leur soient expliqués. Des mots sont proscrits, mais les raisons restent implicites. Ainsi, si l'apprenti se laisse aller à dire "j'adore » ou "j'aime » à propos des bébés, il lui sera seulement rappelé qu'il n'a " pas à aimer les enfants, que ses parents sont là pour cela », laissant dans l'implicite la crainte d'un attachement non professionnel, voire malsain. Est-ce vraiment par la censure de tels mots que la distance professionnelle nécessaire pour prendre en charge la vulnérabilité des tout-petits peut être comprise?

Ces savoirs sur les questions liées à l'attachement sont rarement abordés en formation, sauf de manière injonctive. Ils mettent parfois ces professionnels débutants face à une tension difficilement tenable : comment travailler sans affect pour garder une distance professionnelle, alors que la relation aux enfants, et notamment aux bébés, nécessite de faire une place aux affects pour communiquer avec eux ? Se révèle ici la complexité de la posture à tenir pour exercer ces métiers : être suffisamment en distance pour ne pas se laisser submerger par l'émotion et les sentiments mais rester assez proche et investi pour engager une relation à l'enfant, en tant que personne et non pas seulement objet de soins. Une élève du CAP PE rapporte de son stage cette impression : "Les bébés, c'est I'horreur. On ne fait rien, on regarde comme ça, mais, bon après un moment, ça va! Moi, je m'ennuie ". L'interaction avec le bébé est d'autant plus délicate que la parole articulée faisant défaut, la médiation avec lui passe par la mise à nu de ses propres affects. Cette confrontation à I'enfant et à soi constitue une épreuve délicate à passer, qui remet en question la possibilité de supporter le travail. Bien des professionnels disent d'ailleurs ne «pas supporter le travail avec les bébés ».

La régulation subtile entre trop d'affects ou pas d'affect, et les raisons qui la justifient, se fonde, en formation, sur la nécessité de ne pas favoriser une relation trop étroite avec les enfants car, étant donné leur statut de stagiaire, il faut protéger l'enfant de la séparation, puisque le stagiaire devra partir à brève échéance.

La distance relationnelle demandée est justifiée par le fait que I'apprentie devra quitter la crèche, donc couper les liens affectifs malencontreusement noués avec les enfants. Une des raisons, non explicite, serait que les liens affectifs noués par les enfants avec les professionnels seraient source de concurrence avec leurs parents. L'attachement, puisqu'il n'est que provisoire, ne doit pas être favorisé pour empêcher les peines de la séparation. Les arguments qui fondent ce principe semblent mettre sur un même plan la nature des liens qui se créent entre les enfants et les parents et ceux que les enfants et les professionnels peuvent avoir ensemble. Cette homothétie supposée des 
sentiments, qui n'accorde pas de crédit à l'enfant sur sa capacité à faire ces différences, conduit à rabattre le travail sur une technique relationnelle et à transformer le professionnel en dispensateur de soin pour éviter tout attachement. Une autre crainte, tout autant tacite, serait de voir les professionnels s'attacher aux enfants et en faire "leur » objet sans rester attentifs aux désirs propres de ces derniers.

Dans cette conception où jeux et soins sont synonymes, la place laissée à la relation et au plaisir comme éléments également contributifs du développement peine à être envisagée. Cette affectivité barrée conduit à prohiber de nombreux gestes (un baiser, une caresse, un surnom affectueux...), au nom d'une nouvelle définition $d^{\prime} u n$ métier qui doit contribuer à construire une relation qui ne soit pas surinvestie, positivement comme négativement. Est-ce nécessaire, pour revaloriser ces métiers peu considérés, de transformer la crèche en un espace stérile où les professionnels voient se surajouter à la complexité intrinsèque de leur travail des obligations nouvelles qui rendent son effectivité encore plus difficile?

\section{Conclusion}

Les formateurs des métiers de la petite enfance font face à un double enjeu : d'une part, faire reconnaître ce travail comme une profession à part entière qui requiert des compétences et non pas seulement des qualités liées à I'inné, d'autre part, faire apprendre le travail auprès des enfants qui, par bien des aspects, demeure méconnu. À ces difficultés de reconnaissance et d'apprentissage du travail s'ajoutent celles liées à la légitimité institutionnelle de ces qualifications, dans la mesure où ces deux diplômes, certificat d'aptitude professionnelle petite enfance et diplôme d'auxiliaire de puériculture, ne sont pas délivrés par les mêmes certificateurs. Dans les crèches, la légitimité du ministère des Affaires sociales et de la Santé semble plus forte que celle de l'Éducation nationale où le CAP demeure perçu comme "le diplôme du peuple » (Brucy et al., 2013).

Conçue par des infirmiers, parfois spécialisés en puériculture, et adressée à des professionnels susceptibles d'intervenir dans les milieux hospitaliers, la formation des auxiliaires est fortement orientée vers une approche du soin à prodiguer à de très jeunes enfants hospitalisés (maternités, enfants porteurs de handicaps, protection maternelle et infantile...). Cette prégnance du soin dans la formation est d'autant plus développée qu'elle permet aux formateurs de rapprocher le métier du monde médical reconnu pour ses savoirs et sa technicité. Le " soin » va donc servir aux formateurs des auxiliaires, mais également des CAP PE, qui, pourtant, n'interviennent pas en milieu hospitalier, à construire une représentation plus valorisante du travail. Il permet d'opérer le glissement entre un travail domestique considéré sans compétence, et un véritable travail de prise en charge de la vulnérabilité, qui se réfère au "care », même si, dans les enseignements, le terme n'est jamais employé. Ce soin, conçu pour des enfants bien portants, devient le moyen d'une reconnaissance professionnelle qui fait défaut socialement mais qui ne prend toujours pas appui sur le travail réellement effectué, voire qui I'invisibilise.

Cette tentative de revalorisation à travers le soin, effectuée par la formation, installe une disjonction importante entre les réalités professionnelles de la garde de jeunes enfants, les discours sur ces fonctions et les pratiques formatives. Les efforts effectués pour contribuer à valoriser la professionnalité de ces deux qualifications consistent à faire acquérir un langage d'emprunt qui fait écho à celui du monde du soin, à imposer des entraînements à des gestes professionnels qui ont pour principales fonctions de se différencier des gestes ordinaires et, pour les auxiliaires de puériculture, à travailler la maîtrise des affects de manière à contraindre leur engagement subjectif de telle sorte que leur activité soit subordonnée au seul développement d'un enfant. L'idée que le «bon geste professionnel », pensé en référence aux savoirs enseignés, non contestables car médicalement fondés, ne peut produire que le bon développement de l'enfant, traverse l'ensemble des modalités pédagogiques conçues pour ces jeunes stagiaires durant leur formation. S'y lisent en filigrane des conceptions hygiénistes du monde qui visent à les conformer à des modes d'existence organisés et structurés selon des règles de vie qui s'imposent à eux sans questionnements possibles.

La dimension du sujet, et plus largement de la vie humaine de l'enfant, paraît dès lors très appauvrie. Subordonné aux gestes justes des professionnels, l'enfant dont il est question en formation est un enfant paradoxal : il apparaît parfois comme un enfant avatar animé par des besoins ${ }^{(9)}$ à satisfaire, avec qui il faut parler et

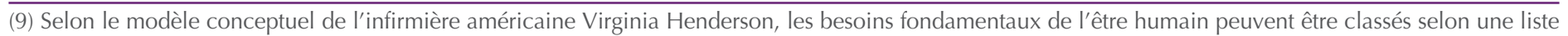
ordonnée, utilisée par les professionnels de la santé. Ces besoins sont appris en formation et permettent d'organiser la démarche de soins.

Revue des politiques sociales et familiales $\mathrm{n}^{\circ} 120-2^{\mathrm{e}}$ trimestre 2015

41 Dossier " Accueil du jeune enfant » 
jouer sans toutefois trop s'engager dans l'échange avec lui ; à d'autres moments, il est déjà un adulte très socialisé, gêné par le regard d'autrui sur sa nudité et qui aurait besoin que l'on préserve « sa pudeur »lors de ses changes. Rarement il est question des dimensions relatives à la corporéité, à la nudité, à l'agressivité des enfants qui s'imposent pourtant dans la réalité. Le travail envisagé en formation, en dépit des nombreuses attentions pour faire reconnaître une professionnalité qui ne se réduit pas à l'activité domestique, demeure " hors sol ", c'est-à-dire très loin des réalités de travail que vont rencontrer ces futurs professionnels. Le langage et le geste constituent dès lors un piège dans lequel le travail réflexif demandé aux professionnels se trouve confiné. Cette réflexivité, essentielle en formation pour comprendre son action et la réajuster en fonction des situations, est réduite ici à des dimensions techniques voire technicistes qui évacuent à la fois le sens et la compréhension de sa posture à l'égard de l'enfant dans la construction de la professionnalité. Sens et distance n'étant pas travaillés dans l'espace de la formation, c'est alors « au projet » de la structure d'accueil que renvoie la formation quand l'enfant rencontré dans les stages ne correspond pas à celui évoqué dans les cours ou les travaux pratiques.

Confrontés à ces ruptures, les professionnels peuvent parfois se retrouver démunis. Il semble dès lors possible d'avancer l'hypothèse que les disjonctions entre les difficultés du travail, les discours et la reconnaissance de ces deux qualifications peuvent constituer des causes non négligeables des forts taux d'absentéisme, et parfois de démissions, dès les débuts de carrière pour cette catégorie de personnels.

- Aballéa F., 2005, La professionnalisation inachevée des assistantes maternelles, Recherches et Prévisions, $\mathrm{n}^{\circ} 80$, p. 55-66.

- Boltansk L., 1971, Les usages sociaux du corps, Les Annales, n 1, p. 205-233.

- Bouve C., Sellenet C., 2011, Confier son enfant. L'univers des assistantes maternelles, Paris, Autrement.

- Brucy G., Maillard F., Moreau G., 2013, Le CAP : diplôme du peuple, 1911-2011, Rennes, Presses universitaires de Rennes.

- Clot Y., 2008, Travail et pouvoir d'agir, Paris, Presses universitaires de France.

- Cresson G., 1998, Formations et compétences dans les métiers de contact direct avec les petits enfants : quelques enjeux, conflits et paradoxes, Lien social et Politiques, n 40, p. 25-37.

- Cresson G., Gadrey N., 2004, Entre famille et métier : le travail du care, Nouvelles questions féministes, $\mathrm{n}^{\circ} 23(3)$, p. 26-41.

- Cru D., 1995, Règles de métier, langue de métier : dimension symbolique au travail et démarche participative de prévention. Le cas du bâtiment et des travaux publics, mémoire de l'École Pratique des hautes études - Laboratoire d'ergonomie physiologique et cognitive, Paris.

- Dejours C., 2009, Travail vivant, Paris, Payot.

- Fagnani J., 2001, La politique d'accueil de la petite enfance en France : ombres et lumières, Travail, genre et sociétés, $\mathrm{n}^{\circ} 2(6)$, p. 105-119.

- Falzon P., 1987, Langages opératifs et compréhension opérative, Le travail humain, n 50(3), p. 281286.

- Gardin B., 1995, Le sens comme production sociale, in Boutet J. (dir), Paroles au travail, Paris, L'Harmattan, p. 151-164. 
- Kergoat D., 1995, La reproduction et le changement, place de la parole, in Boutet J. (dir.), Paroles au travail, Paris, L'Harmattan, p. 165-180.

- Laufer J., Marry C., Maruani M., 2003, Le travail du genre : les sciences sociales du travail à l'épreuve des différences de sexes, Paris, La Découverte.

- Laugier S., 2006, Care et perception. L'éthique comme attention au particulier, in Paperman P., Laugier S. (dir.), Le souci des autres. Éthique et politique du care, Paris, Éditions de l'ÉHESS. p. 315-348.

- Leplat J., 2013, Les gestes dans l'activité en situation de travail. Aperçu de quelques problèmes d'analyse, Perspectives interdisciplinaires sur le travail et la santé, nº 15(1), mis en ligne le 18 février 2013, URL://pistes.revues.org/2951.

- Marchand Montanaro G., 2013, Travailler en crèche, un métier?, Rennes, Presses de l'École des hautes études en sciences politiques.

- Maruani M., Meron M., 2012, Un siècle de travail des femmes en France : 1901-2011, Paris, La Découverte.

- Murcier N., 2007, La réalité de l'égalité entre les sexes à l'épreuve de la garde des jeunes enfants, Mouvements, $n^{\circ} 1(49)$, p. 53-62.

- Murcier N., 2004, De la difficulté à se représenter l'enfant sexué, La lettre de l'enfance et de l'adolescence, $\mathrm{n}^{\circ} 4$, p. 89-95.

- Odena S., 2009, Les professions et leur coordination dans les établissements d'accueil collectifs du jeune enfant : une hétérogénéité source de tensions au sein des équipes. Dossier d'études, Caisse nationale des Allocations familiales, $n^{\circ} 121$.

- Rayna S., 2011, Profils et formation des personnels de la petite enfance au regard du bien-être des enfants : spécificités et ouverture, in Bien-être des jeunes enfants dans l'accueil et l'éducation en France et ailleurs, actes du colloque des 10 et 11 octobre, direction de la recherche, des études, de l'évaluation et des statistiques - Centre d'analyse stratégique, Collection études et statistiques, p. 75-89.

- Schwartz Y., 1988, Expérience et connaissance du travail, Paris, Liaisons sociales.

- Tronto J. C., 1993, Moral Boundaries: A political argument for an ethic of care. New York:Routledge, traduction française par Maury H., Un monde vulnérable. Pour une politique du care, Paris, La Découverte, 2009.

- Ulmann A.-L., Betton, E., Jobert, G., 2011, L'activité des professionnelles de la petite enfance, Dossier $d^{\prime}$ études, Caisse nationale des Allocations familiales, $\mathrm{n}^{\circ} 145$.

- Verba D., 2014, Le métier d'éducateur de jeunes enfants, un certain regard sur l'enfant, Paris, La Découverte.

- Vigarello G., 2014, Le propre et le sale. L'hygiène du corps depuis le Moyen Âge, Paris, Le Seuil.

- Zogmal M., 2008, "T'es un enfant à caprices ». Les stratégies défensives du métier d'éducatrice du jeune enfant, Cahier de la section des sciences de l'éducation, $n^{\circ} 119$. 